\title{
Autokinesis direction during and after eye turn
}

\author{
JOHN LEVY* \\ Columbia University, New York, New York 10027
}

\begin{abstract}
The aftereffect (AE) of eye turn on autokinesis direction is usually, but not always, opposite to the inducing turn direction. During four experiments, a model predicting the aftereffect's time course and a new measure utilizing the cancept of the position of random autokinetic movement (PRAKM) were developed. They showed that aftereffect direction alternates during dissipation and that its first direction is not a simple function of previous eye position, but of the process by which that position is achieved, suggesting that at least two processes are involved. In one $S$, versions produced the usual $\mathrm{AE}$, while, after vergences, the $\mathrm{AE}$ was in the same direction as the inducing turn. Differential recruitment of these systems in monocular fixation could account for individual differences in the AE.
\end{abstract}

A stationary spot of light, viewed monocularly in the dark immediately following an eye turn, is usually described as moving in the same orientation as, and mostly opposite to, the direction of the preceding eye turn, then reversing direction, perhaps several times (Gregory \& Zangwill, 1963). Levy (1972), who has reviewed the autokinetic illusion (AKI), found that the most intriguing theoretical problems and potentially fruitful area for future research involved this aftereffect (AE) of eye turn on AKI direction, since what was then known about it could not be explained by any theory. He argued that the AKI was primarily due to involuntary small drifts caused by consistent mechanical forces in the extraocular muscles. However, eye movements correlated with AKI direction did not always occur. To make the theory consistent with this fact in both the AKI and many related illusions of apparent movement, it was necessary not only to apply the outflow theory (Merton, 1964), but to assume that pursuit eye movements were also outflow-monitored. Even this expanded outflow model assumes that the extraocular forces occurring at any one eye position are fixed. It cannot explain the $A E$, where a fixed AKI stimulus appears to go in various directions as a function of the previous eye position. Slowly dissipating, temporary changes in oculomotor balance must occur, although they cannot be predicted from the mechanisms included in any theory.

Carr (1910), who first recognized that some such process must occur, invoked the concept of "strain" or "fatigue," but Gregory and Zangwill (1963) point out that this implies that the muscle's power is weakened so that the eye will drift away from the fatigued side. Consequently, if the drift is undetected, the resulting apparent movement should appear to be towards the fatigued side. Since they found that the opposite $\mathrm{AE}$ is more common, they suggested that changes in the "monitoring" or "command" systems were more likely than were changes in the muscle. However, there is a

*Present address: Department of Applied Psychology. University of New South Wales. P.O. Box 1. Kensington, N.S.W., Australia 2033. third possibility, i.e., that muscle tension actually remains higher after prolonged contraction than it does in the resting state. This effect certainly occurs in isolated nerve/muscle preparations in the phenomenon of posttetanic potentiation (Lloyd, 1949). It can also be simply demonstrated in vivo by any isometric exercise. such as holding a weight straight out from the body. When relieved, the arm moves up as the strained muscles continue to contract.

Any process causing tension increase following contraction can, when added to Levy's (1972) expanded outflow theory, explain not only why the first $\mathrm{AE}$ direction is usually opposite to that of the preceding eye turn, but also why it often reverses cyclically as Gregory and Zangwill (1963) report. Since the agonist is excessively tense after contraction, the eye will drift towards it. This unmonitored drift causes apparent movement. Pursuit-like outflow to the antagonist will increase so as to stabilize fixation. The antagonist in turn becomes overtense as the effect in the agonist dissipates. The developing effect in the antagonist will then itself be excessive, causing drift and reversing the AKI. Several such alternations could occur before normal balance was restored.

Data already available suggested that the prediction about the first direction of the AE would not always be confirmed. Carr (1910) and Gregory and Zangwill (1963) both found some Ss who reported AEs in the same direction as the inducing eye turn following some angles of eye turn and various stimulation and dissipation times. Levy (1968) reports the first two parametric studies of the $\mathrm{AE}$. In the first, all $11 \mathrm{Ss}$ showed clear AEs in the direction opposite to that of the previous turn, but in the second. with only minor improvements of technique, there was no significant main effect and variability over Ss was well outside the range of normal error.

\section{EXPERIMENT I}

Levy's (1968) experiments on the AKI had required developing an adequate measure of it. He argued that. 
because of the phenomenon of paradoxical movement, direction reports were the only theoretically relevant, unambiguous AKI responses that could be used. When the AKI stimulus is straight ahead. one reported direction predominates in each eye and this bias is reliable over long periods (Pearce. 1966). When stimuli are fixated at other positions, the likelihood that the AKI will be reported going in the direction of eye turn increases as a function of angle. It is therefore possible to define the position of random autokinetic movement (PRAKM) as that stimulus position where, if binary forced-choice movement direction reports are demanded from an $\mathrm{S}$ monocularly viewing a stationary spot in the dark, either response is equally likely (Levy, 1968, 1972). Levy described a staircase technique by which he could show PRAKM to be a reliable measure, unique in each eye, determinable to within a standard error of 3-5 deg. which remained stable for periods of over a week and which was in 10 cases out of 12 identical to the physiological position of rest.

To indicate AE size, his first two parametric studies of AKI AE used the degree to which PRAKM was shifted when each stimulus was preceded by an eye turn. Following classical AE studies, he alternated an inducing figure (IF), in this case a spot of light at a fixed position which $\mathrm{S}$ fixated, with a test figure (TF). an identical spot of light, fixated at a position which varied after each judgment in accordance with a staircase rule. The results were inconclusive, possibly due to sources of error, including the variety of TF positions used in this "PRAKM shift" method.

This experiment is a trial of a new method. It is reported because of the theoretical importance and unexpected nature of the result. It was based on the idea that the AKI AE's direction could be directly related to a shift in response probability if the TF was always at PRAKM. This method has many advantages: only two stimulus positions, the IF and TF, are alternated; eye position is thus fully controlled; and there is complete flexibility in stimulation and response times. If responses are initiated by a sound and many responses are demanded at fixed intervals throughout each cycle, it becomes possible to test the prediction. derivable from the above theoretical account, that the time course of probabilities of binary forced-choice AKI direction reports to a stimulus at PRAKM, following eye turn, will be first in the opposite direction to the preceding eye turn, then alternating and fading like a dampened sinusoid.

\section{Method}

\section{Apparatus}

A dim light of .01-in. diam was set on a long bar, mounted relative to a biteboard in such a way that it could be rotated through either the horizontal or vertical planes to any point on the circumference of a sphere whose center was the center of rotation of $S$ 's eye and whose radius was normally $5 \mathrm{ft}$. It was the stimulus for the pretest and the TF in the AE phase. An identical light source, on a fixed stand. served as the IF stimulus in the AE phase. The $S$ sat on a stool, his head held by the biteboard and his left eye occluded. He responded by throwing a switch in the direction he wished to report. It could be positioned for either up/down or left/right alternatives and displayed a red or green light near $\mathrm{E}$, who sat behind $\mathrm{S}$ and recorded his responses using a dim light which did not illuminate the dark room. In the pretest phase. $E$ initiated each 240 -sec stimulus presentation by pressing a button, which started a set of timers in the next room. In the AE phase, he selected and started a longer sequence, which continuously alternated 180 -sec IF and 240-sec TF and produced a short buzz every $5 \mathrm{sec}$ throughout the TF phase, until interrupted. Then $E$ had only to record S's responses.

\section{Subjects}

The first S (M.C.) normally wore reading glasses, but did not need or use them in this situation. She was without any previous experience in psychophysical experiments and had no knowledge of the hypothesis being tested. She later acted as $\mathrm{E}$ while the author (J.L.) was S.

\section{Procedure}

After a biteboard had been made and $S$ fitted into the apparatus, she was instructed for the pretest phase. She had to fixate each stimulus all the time it was present and, when it went off, to report whether its final direction of movement was left or right, then to keep looking in the same direction until the next stimulus onset. In the dark period of approximately $10 \mathrm{sec}, \mathrm{E}$ recorded S's response, selected the next stimulus in accordance with the stepping rules of Taylor and Creelman's (1967) PEST technique, and turned on the stimulus. The staircase started with 10-deg steps and stopped when a 1-deg step was required. This final stimulus position defined PRAKM and was the TF position in the AE phase. PRAKM was -22 deg horizontal $(H)$ for M.C. and 0 deg $\mathrm{H}$ for J.L. 1 Only the horizontal AE and horizontal PRAKM were studied in this experiment. The vertical (V) position of all stimuli was always $0 \mathrm{deg} V$.

In the $\mathrm{AE}$ phase, $\mathrm{S}$ was told to fixate each light as long as it was present and to report its movement direction each time the buzzer sounded. Only two IF positions $( \pm 40 \mathrm{deg} \mathrm{H})$ were used. These were close to the largest possible eye turns and were expected to produce a large AE. Similarly, the stimulation and dissipation times were chosen because they were sufficient for other body-position AEs to develop fully and dissipate appreciably (Singer \& Day, 1965). These parameters suited the exploratory nature of the study. Once $E$ had started the cycle, he had only to record S's responses to the TF. Because each cycle was $7 \mathrm{~min}$ long, it took three or four sessions to collect enough data for each $\mathrm{AE}$ curve, e.g., 20 repetitions. The Ss halted whenever they needed to. The data from the first cycle after each rest was rejected in order to reduce any possible variance. Afterwards, the frequency of right responses in each $5-\mathrm{sec}$ interval was summed and plotted.

\section{Results and Discussion}

The resulting curves are shown in Fig. 1. To make this data consistent with the later experiments, the \pm 40 -deg H IF positions are specified relative to PRAKM rather than $0 \mathrm{deg} \mathrm{H}$. In three of the four curves, there is a clear first effect in the opposite direction to eye turn followed by at least two reversals. ${ }^{2}$ It is not clear whether the alternation has finished after the $4 \mathrm{~min}$. In the fourth case (M.C.. IF = PRAKM $+62 \mathrm{deg} \mathrm{H}$ ). the 


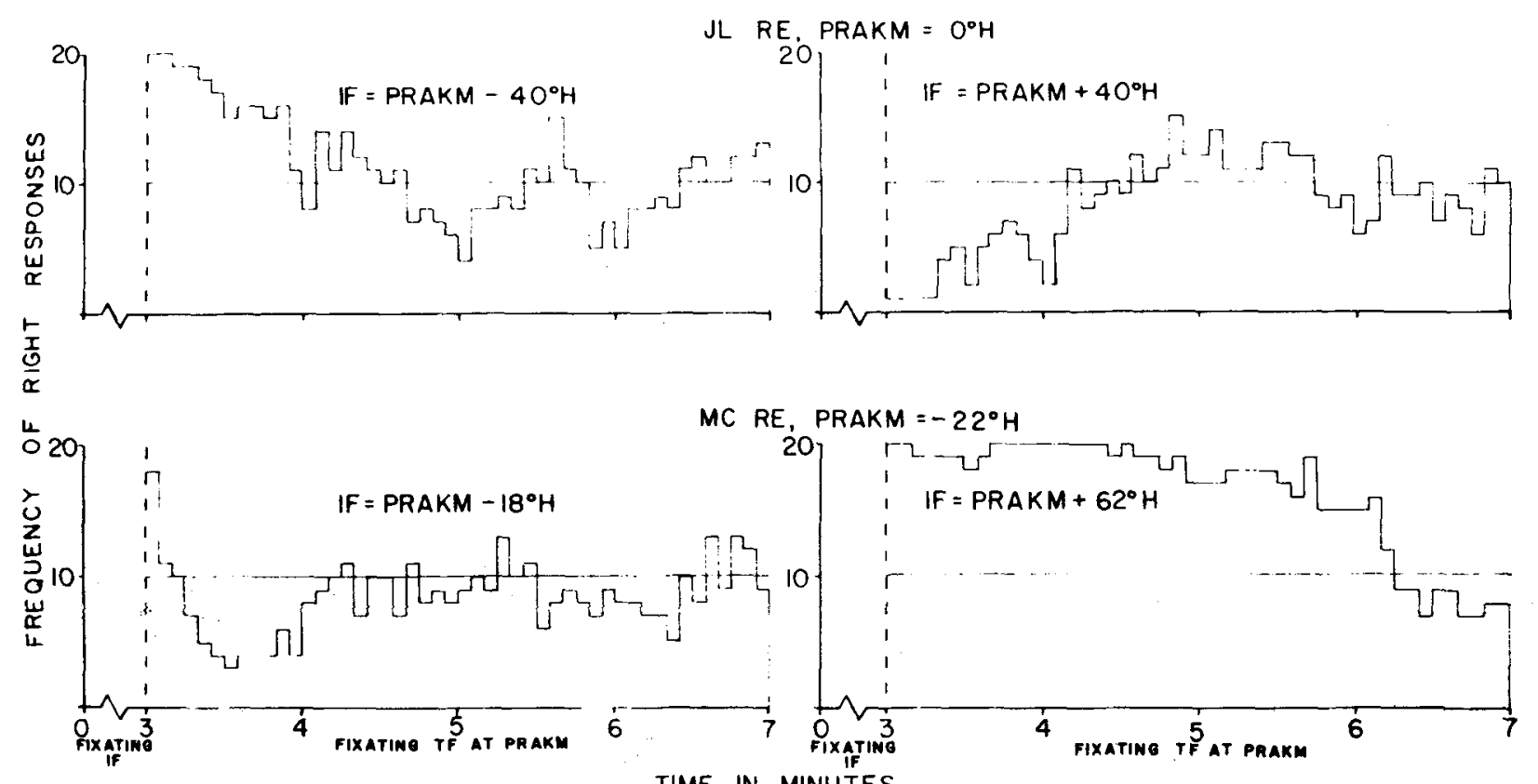

Fig. 1. Histograms of cumulative right response frequency over time for two Ss in Experiment I. J.L.'s right-eye PRAKM = 0 deg H, M.C.'s $=-22 \mathrm{deg} H$.

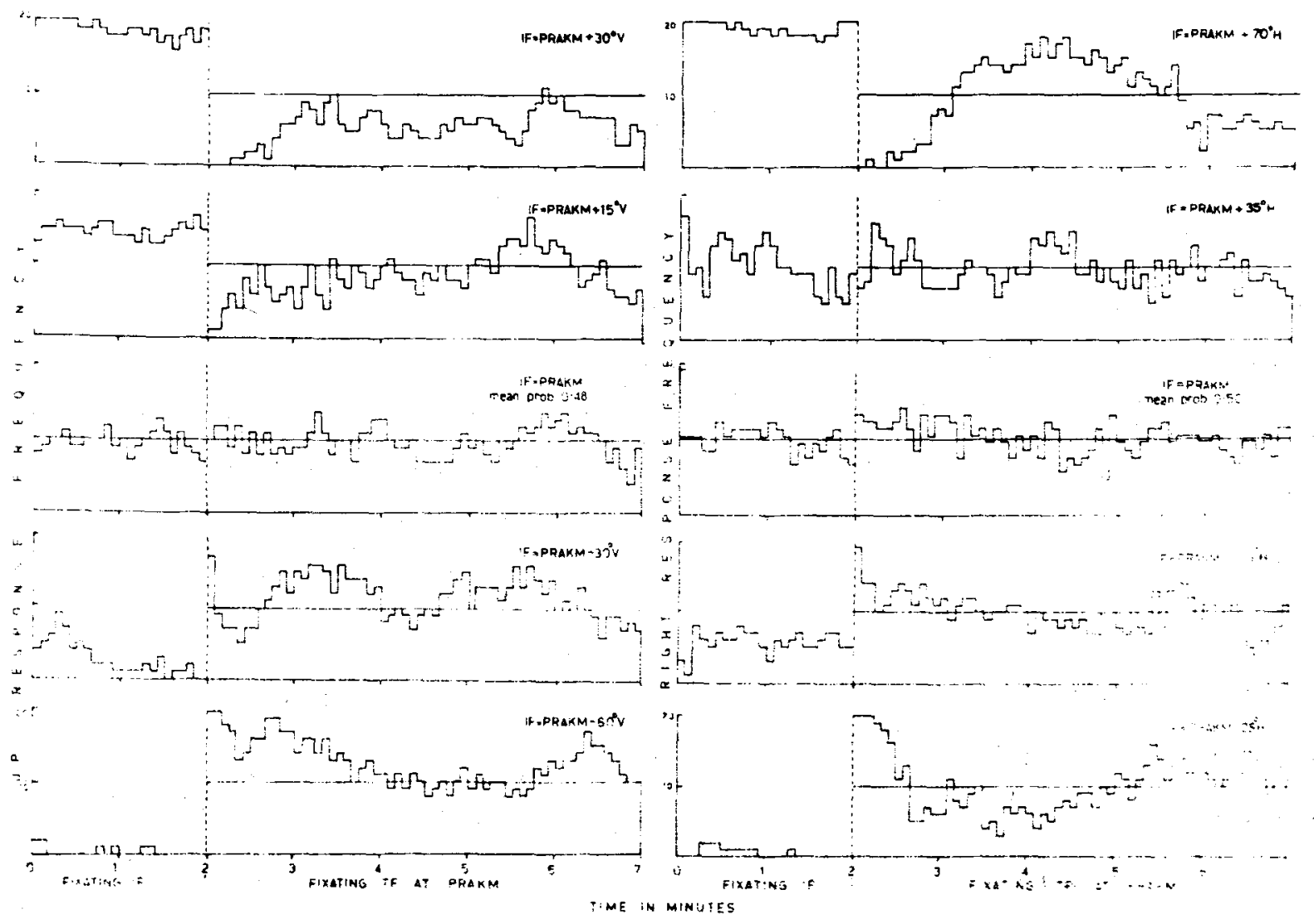

Fig. 2. Histograms of cumulative right or up response frequency over time for A.M. in Experiment II. A.M.'s right-eye PRAKM = $-22 \mathrm{deg} \mathrm{H}, 17 \mathrm{deg} \mathrm{V}$ at $5 \mathrm{ft}$. 


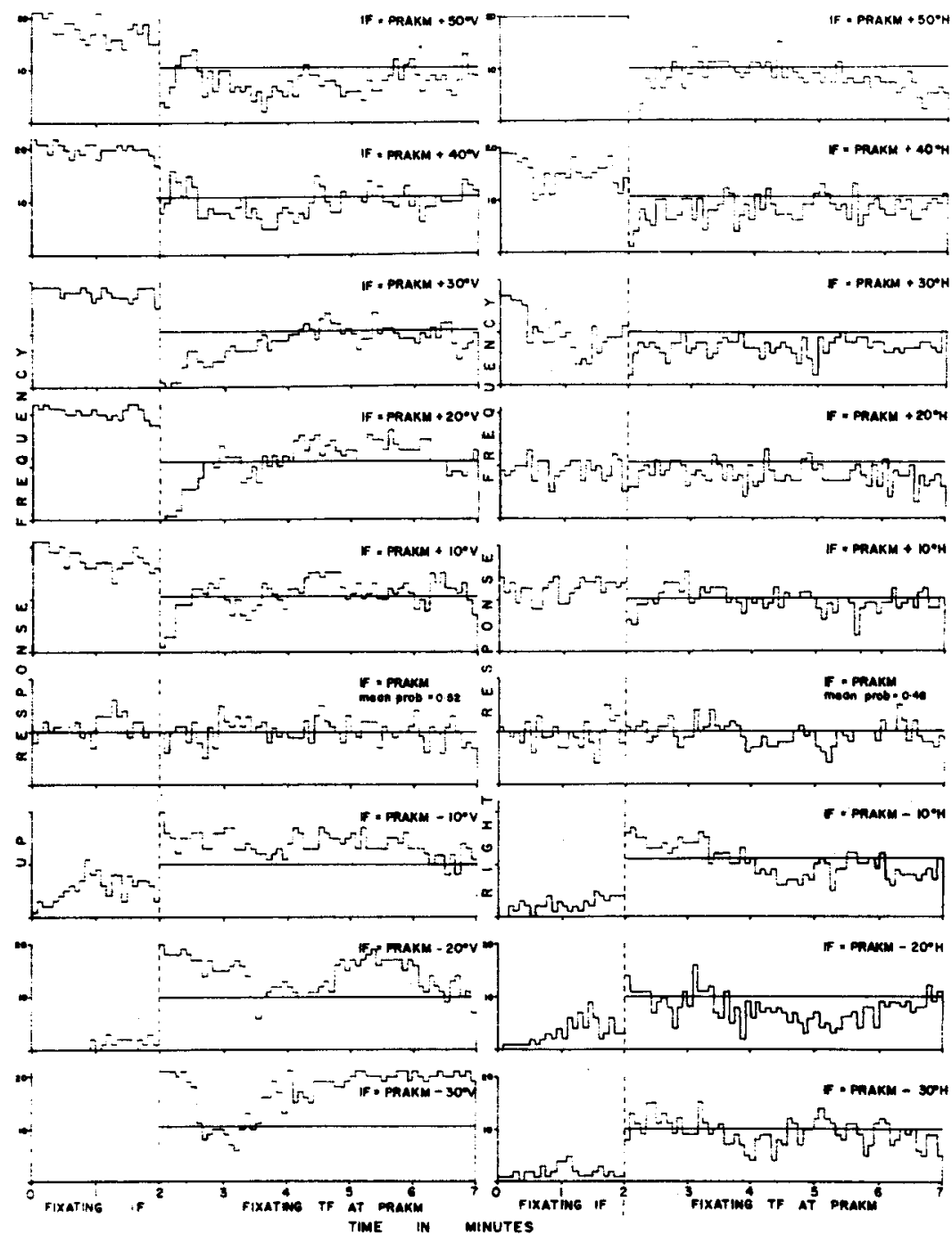

Fig. 3. Histograms of cumulative right or up response frequency over time for J.L. in most conditions of Experiment II. J.L.'s righteye PRAKM = $-6 \operatorname{deg} \mathrm{H},-17 \mathrm{deg} \mathrm{V}$ at 5 ft.

opposite effect occurs so strongly that it just reaches its first reversal before recycling. Clearly, this is an unpredicted result. Its explanation is a major theoretical problem. In practical aspects, the method needed only a little improvement.

\section{EXPERIMENT II}

This study explored the AE systematically, using IF positions over the whole horizontal and vertical ranges of two Ss' visual fields. It was hoped that some pattern might be found which could be related to the reversals of some Ss' AEs.

\section{Method}

A few changes were made in the previous procedure; the TF fixation time was increased by $60 \mathrm{sec}$ so that more AE dissipation would occur. Unfortunately, IF fixation was reduced by a like amount because 7 min was the maximum recycle time possible; automatic stimulus and response recording made it possible for Ss to run themselves in the AE phase. PRAKM was measured in both dimensions. When the $\mathrm{AE}$ in one dimension was studied, the stimuli were placed along the arc which passed through PRAKM on the orthogonal dimension too, thus making the effect of interest clearer. In vertical PRAKM and AE measures, Ss naturally chose between up or down movement reports; responses to the IF were also demanded in order to ensure that $S$ maintain fixation and that the eye-turn effect be normal, even when the AE was not.

For the author (J.L.), PRAKM was remeasured by an independent $\mathrm{E}$ and found to be $-6 \mathrm{deg} \mathrm{H},-17 \mathrm{deg} \mathrm{V}$. His $\mathrm{AE}$ functions were collected by using IF figures spaced every $10 \mathrm{deg}$ across the whole visual range (PRAKM +50 deg $\mathrm{H}$ to PRAKM $-50 \mathrm{deg} \mathrm{H}$, PRAKM $+70 \mathrm{deg} \mathrm{V}$ to PRAKM $-50 \mathrm{deg} \mathrm{V})$. The functions so obtained are shown in Figs. 3 and $4 .{ }^{3}$

Since M.C. was not available, another naive female $S$ (A.M.) was used next. Her PRAKM was $-22 \mathrm{deg} H, 17 \mathrm{deg}$ V. Ten IF positions were used to cover the range of possible eye turns from the most extreme to PRAKM, with one intermediate point in each cardinal dimension (PRAKM +70 deg $\mathrm{H}$ to PRAKM $-25 \mathrm{deg} \mathrm{H}$, PRAKM $+30 \mathrm{deg} \mathrm{V}$ to PRAKM $-60 \mathrm{deg} \mathrm{V}$ ). To further ensure that regularities did not result from maintained habits, her 20 trials with each IF were broken into 4 blocks of 6 and the resulting 40 blocks run in a randomized order. The obtained functions are shown in Fig. 2. 


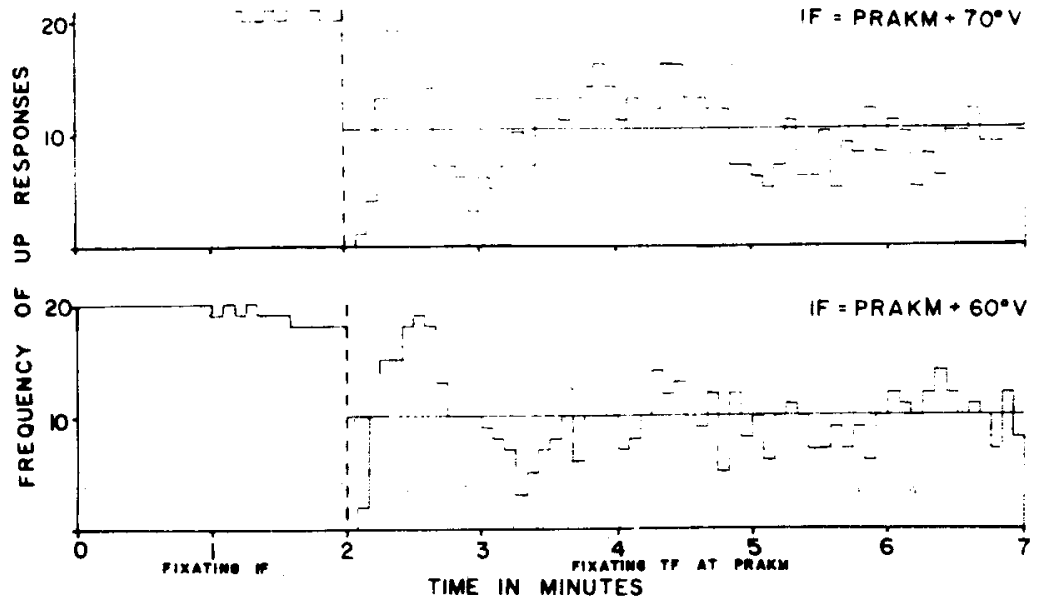

Fig. 4. Histograms of cumulative up response frequency over time for J.L. with extreme upper vertical IF stimuli in Experiment II. J.L.'s right-eye PRAKM = -6 deg $H,-17$ deg $V$ at 5 ft.

\section{Results}

The eye-turn effect of the IF shown in the first 2 min of each graph is the expected function of angle. Its intensity generally decreases with fixation time, indicating that adaptation is occurring. The nonlinear relation to angle seen in J.L.'s horizontal effect, indicating that there is little eye-turn effect over a wide range, can be correlated with his generally depressed horizontal AE.

The AE data from A.M. (the last 5 min of each curve in Fig. 2) fits the predictions fairly closely. However, at the vertical extremes, the reversals, in the same direction as eye turn, do not go as far beyond the $50 \%$ base line as would be expected, suggesting that the $\mathrm{AE}$ has two components, one dissipating more slowly than the process which causes the oscillation. The horizontal effect is much less intense and, after the moderate turns, stabilizes quickly.

These same features are seen even more clearly in J.L.'s data (Figs. 3 and 4). The vertical effect particularly seems to be composed of an exponentially decreasing function overlaid by alternations. The initial size and dissipation time of the exponential, as well as the intensity and rate of onset of the first reversal, increase with angle. J.L.'s horizontal effect and $\mathrm{AE}$ are both very suppressed. The most important feature is that the $\mathrm{AE}$ decreases with angle of left-eye turn, contrary to expectations. Symmetrically, the right-eye-turn effect is reduced, as was noted above. These unexpected features suggested that the $\mathrm{AE}$ involves more complex processes than the model postulated.

\section{Discussion}

The unexpected AEs had been mostly in the horizontal. Subjective impressions also confirmed that the horizontal effect was weaker and more difficult to judge, especially late in the AE. Despite all the controls, vertical movements predominated.

In maintaining a given monocular horizontal eye turn. either the version or vergence system might be recruited to varying degrees. The unexpected features of horizontal AEs could result from individual differences in the weight given to each mechanism and to differing directions of action during both IF and TF fixation. There are large individual differences in their interaction pattern when the eyes are unfused, as shown by the many functions of AC/A ratio possible (Alpern, 1962). Ludvigh $(1952 a, b)$ presents evidence to suggest that only versions are outflow-monitored.

If convergence did function differently, the direction of the AE arising from holding the eye at any position should vary as a function of the mechanism used to achieve it. The original model assumes that each muscle acts independently and that the $\mathrm{AE}$ is a simple consequence of tonus tending to remain at the preturn level. It implies that the $\mathrm{AE}$ at any position should always be the same, no matter which mechanism is used to turn the eye. The next experiment was a simple test of these opposite predictions.

\section{EXPERIMENT III}

\section{Method}

The only $S$ in this experiment was J.L. The IFs used in this experiment came from the same light sources as before, but they were viewed binocularly at small distances with symmetrical binocular convergence so that the angles formed between each line of sight and the median plane were 6,16 , or $26 \mathrm{deg}$. Thus, J.L.'s right eye was in exactly the same positions as at PRAKM, PRAKM $-10 \operatorname{deg} \mathrm{H}$, and PRAKM $-20 \mathrm{deg} \mathrm{H}$, respectively. The occluder was changed so that the IF was viewed binocularly and the TF by the right eye only. The TF remained at PRAKM ( $-6 \operatorname{deg} \mathrm{H},-17 \operatorname{deg} \mathrm{V})$ and $5 \mathrm{ft}$ away, as in the previous experiment. The IF showed no apparent movement, nor was any expected, since with close binocular fixation each monocular eye-turn effect would be opposite and approximately equal, cancelling each other out. Therefore, no direction reports were collected during II fixation. Otherwise, the procedure with the three stimuli was the same as it had been for J.L. in the previous experiment. 


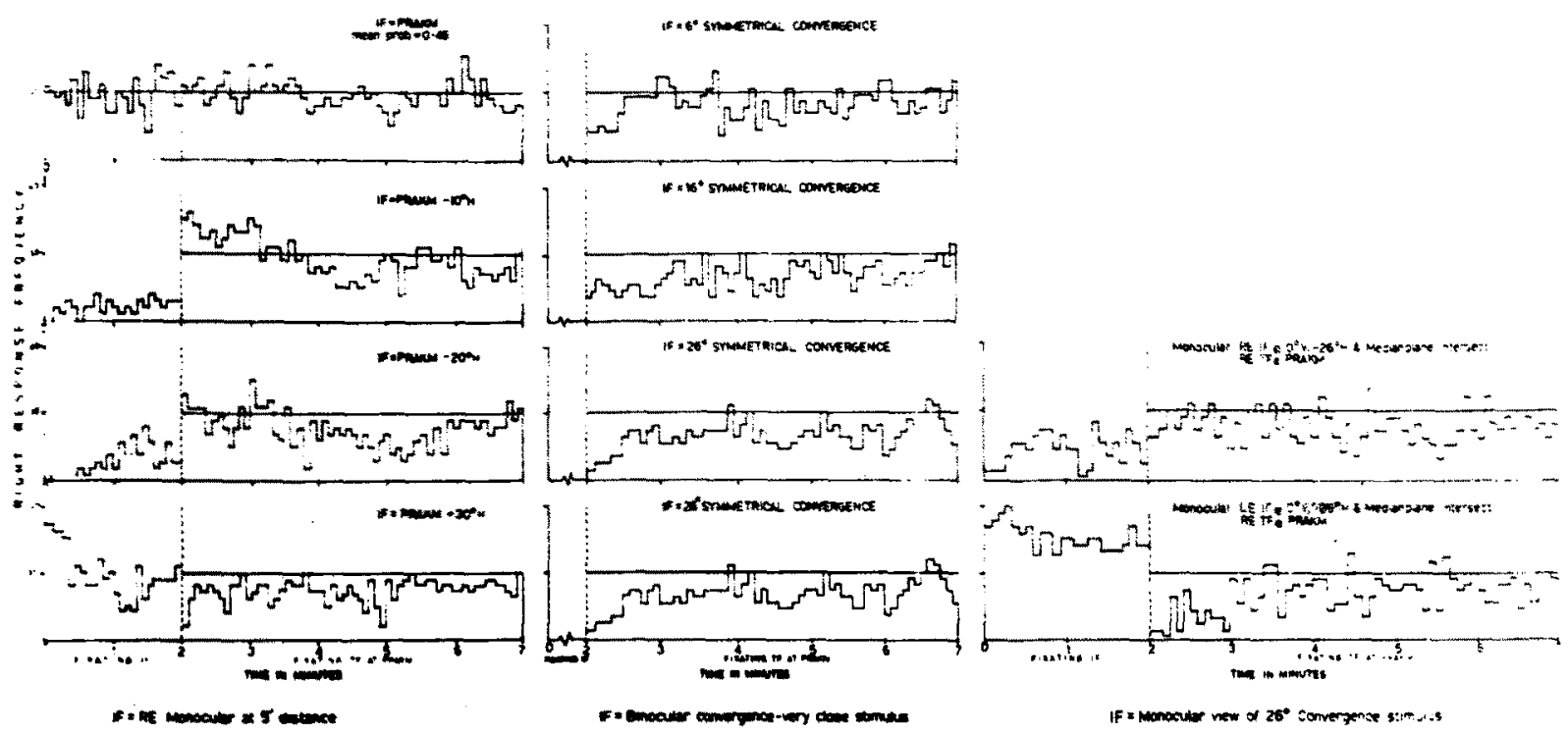

Fig. 5. Comparison of histograms of cumulative right response frequency over time for J.L. when specific right-eye positions (rows) are achieved by various processes (columns). Column 1 contains results from Experiment II (versions), Column 2 the results of Experiment III (convergences), and Column 3 the results of Experiment IV (versions and accommodation/vergence). J.L.'s right-eye PRAKM $=-6 \operatorname{deg} \mathrm{H},-17 \mathrm{deg} \mathrm{V}$ at $5 \mathrm{ft}$.

\section{Results}

The results in the second column of Fig. 5 show clearly that when the previous eye turn is achieved by convergence, the $\mathrm{AE}$ goes in the same direction as the previous turn, and this effect increases with angle. Subjective reports confirm the pattern of the graphs, which show that the first phase of these AEs was the most intense so far experienced. In the first few seconds, the movement was so fast that fixation could not be maintained.

\section{Discussion}

We can conclude that the direction of the AKI aftereffect has no fixed relation to any given preceding eye position, but is a function of the differential weight of the mechanisms by which the preceding IF fixation is achieved. Usually, vergences produce AEs in the same direction as the preceding eye turn and versions to AEs in the opposite direction. After the first minute, the convergence effect remained at a set level which increased with inducing angle. This slowly dissipating component resembles that noted in the last experiment, except that it is in the opposite direction. The small degree of dissipation after 5 min also marks this as an exceptional AE.

\section{EXPERIMENT IV}

\section{Method}

Since convergence, accommodation vergence, and left-eye TF fixation contributed to the above AE. it was decided to see what the function was of the latter two alone. Again, J.L. was the only S. Accordingly, data were collected for the AEs which resulted when the 26-deg convergence IF stimulus used in the last experiment was fixated monocularly by each eye. The TF fixation was exactly as usual. Occlusion was modified so that the appropriate fixation occurred at each phase, but the experimental situation was otherwise unchanged. The resulting functions are shown in the last column of Fig. 5 .

\section{Discussion}

The eye positions achieved can be regarded as a combination of version and vergence effects. By comparing the AEs with those which would have resulted if either mechanism had acted alone, we can see how the effects interact. The right-eye IF fixation could have been achieved by a PRAKM -20-deg $\mathrm{H}$ version, as in Experiment II, or a 26-deg convergence, as in Experiment III. The AEs resulting in these three cases are compared in the third row of Fig. 5. Since the first two are in opposite directions, the third, lying midway between them, is just what would be expected if it were a weighted sum of the other two. Similarly, with a +26-deg $H$ left-eye turn, a pure version would be paralleled in the right eye, so we would expect an $\mathrm{AE}$ similar to that following a $+26-d e g ~ H$ right-eye turn, i.e., PRAKM $+32 \operatorname{deg} \mathrm{H}$, for which the nearest available comparison is PRAKM $+30 \mathrm{deg} \mathrm{H}$. If pure vergence were used, the same effect as before would be expected. The three alternatives, shown in the bottom row of Fig. 5, again suggest summation. The slowly dissipating vergence effect is clearly seen in both graphs collected in this experiment. 


\section{SUMMARY AND CONCLUSION}

These studies of the $\mathrm{AE}$ of eye turn on $\mathrm{AKI}$ direction were stimulated by the fact that the $\mathrm{AE}$ is the one AKI phenomenon which cannot be accounted for by any theory. Even the most comprehensive model (Levy, 1972) did not include a mechanism by which slowly dissipating temporary changes in oculomotor balance might occur. Although the model was complicated by increasingly complex potential mechanisms, the experimental results were always more complex than those predicted by the theory. The most puzzling feature was the complete reversals of the $\mathrm{AE}$ direction found in some cases. Experiments I and II showed that more than a simple potentiation of previously contracted muscles was involved, while Experiments III and IV showed that the AE direction was not a function of the previous eye position at all, but rather of the process by which that eye position had been reached, versions producing $A E s$ in the opposite direction to that of the previous turn and vergences to AEs in the same direction. Individual differences in $\mathrm{AE}$ direction can then be accounted for by differential recruitment of the two processes during monocular fixation.

This does not mean that the idea that orbital imbalance causing drifts or compensatory pursuit-like outflow is the primary cause of the AKI should be abandoned in favor of a "central" theory, but rather that the model is still too simple to predict the direction of tonic imbalance induced in these special cases. The mechanisms postulated so far no doubt exist and are involved. There are others, such as the role of outflow monitoring (Ludvigh, $1952 \mathrm{a}, \mathrm{b}$ ) and spindle feedback (Breinin, 1957; Baichenko, Matyushkin, \& Suvrov, 1968), which must also be considered.

Alpern (1962) argues that the version and vergence systems remain distinct even within the extraocular muscles. All the above results could be explained if, for example, only versions acted via the spindles and led to potentiation, while vergences acted directly on the muscle fibers and led to fatigue.

\section{REFERENCES}

Alpern, M. Movements of the eyes. In H. Davson (Ed.), The eye. Vol. 3. New York: Academic Press, 1962.

Baichenko, P. I., Matyushkin, D. P., \& Suvrov, V. V. Participation of fast and tonic oculomotor systems in stretch reflexes and labyrinthine reflexes of extraocular muscles. Neuroscience Translations, 1968, 3. 350-358.

Breinin, G. M. Electromyogravic evidence for ocular muscle proprioception in man. Archives of Ophthalmology, 1957, 57. $176-180$

Carr, H. A. The autokinetic sensation. Psychological Review, $1910,17,42-75$.

Gregory, R. L., \& Zangwill, O. L. The origin of the autokinetic effect. Quarterly Iournal of Experimental Psychologyr, 1963. $15,252-261$.

Levy, J. L. The measurement and explanation of the directional component of the autokinetic illusion. Unpublished MS thesis. Monash University, 1968.

Levy, J. L. The autokinetic illusion: A critical review of measurement, theories, and independent variables. Psychological Bulletin, 1972, 78, 457-474.

Lloyd, D. C. P. Post-tetanic potentiation of response in monosynaptic reflex pathways of the spinal cord. Journal of General Physiology, 1949, 33, 147-170.

Ludvigh, E. J. Possible role of proprioception in the extraocular muscles. Archives of Ophthalmology, 1952a, 48, 436-441.

Ludvigh, E. J. Control of ocular movements and visual interpretation of environment. Archives of Ophthalmology. $1952 \mathrm{~b}, 48,442-448$.

Merton, P. A. Absence of conscious position sense in the human eyes. In M. A. Bender (Ed.), The oculomotor system. New York: Harper \& Row, 1964. Chap. 14.

Pearce, D. G. Consistency of individual patterns of autokinetic direction. Perceptual \& Motor Skills, 1966, 23, 1119-1123.

Singer, G., \& Day, R. H. Temporal determinants of a kinesthetic after-effect. Journal of Experimental Psychology, 1965, 69, 343-348.

Taylor, M. M., \& Creelman, C. D. PEST: Efficient estimates on probability functions. Journal of the Acoustical Society of America, 1967, 41, 782-787.

\section{NOTES}

1. In accordance with convention, positive sign refers to angles above or to the right of the reference point, which is, unless otherwise labeled, straight ahead of S's eye. Horizontal or vertical deviation is indicated by the letters $\mathrm{H}$ or $\mathrm{V}$, respectively. Since it is more theoretically relevant. IF positions are fixed relative to PRAKM.

2. Tests of significance are difficult. From the binomial theorem, the probability of any one 5-sec column differing from the mean by more than \pm 4 by chance is 0.02 . When a set of columns do go together, smaller departures over longer times become significant, but, since grouping is arbitrary, such statistics are illegitimate. It is preferable to consider only broad trends which are so obvious that no tests of significance are necessary.

3. Sometimes, for both Ss, data of extra trials were collected. When this happened, the extra data were included. The actual number of trials in any graph is shown by the height of the ordinate.

(Received for publication August 30, 1971;

first revision received February 21, 1972:

second revision received November 23, 1972.

Delay due to the author's moving to Sydney, Australia.) 